\title{
Funnel-freezing versus heat-stabilization for the visualization of metabolites by mass spectrometry imaging in a mouse stroke model
}

\author{
Inge A. Mulder ${ }^{1 *}$, Clara Esteve ${ }^{2 *}$, Marieke J.H. Wermer ${ }^{1}$, Mathias Hoehn $^{3,4,5}$, Else A. Tolner ${ }^{1,6}$, \\ Arn M.J.M. van den Maagdenberg ${ }^{1,6}$ and Liam A. McDonnell ${ }^{2,7}$ \\ ${ }^{1}$ Department of Neurology, Leiden University Medical Center, Leiden, The Netherlands \\ ${ }^{2}$ Center for Proteomics and Metabolomics, Leiden University Medical Center, Leiden, The Netherlands \\ ${ }^{3}$ Department of Radiology, Leiden University Medical Center, Leiden, The Netherlands \\ ${ }^{4}$ Percuros BV, Enschede, The Netherlands \\ ${ }^{5}$ In-vivo-NMR Laboratory, Max Planck Institute for Neurological Research, Cologne, Germany \\ ${ }^{6}$ Department of Human Genetics, Leiden University Medical Center, Leiden, The Netherlands \\ ${ }^{7}$ Fondazione Pisana per la Scienza ONLUS, Pisa, Italy
}

Tissue preparation is the key to a successful matrix-assisted laser desorption/ionization (MALDI) mass spectrometry imaging (MSI) experiment. Rapid post-mortem changes contribute a significant challenge to the use of MSI approaches for the analysis of peptides and metabolites. In this technical note we aimed to compare the tissue fixation method ex-vivo heat-stabilization with in-situ funnel-freezing in a middle cerebral artery occlusion (MCAo) mouse model of stroke, which causes profound alterations in metabolite concentrations. The influence of the duration of the thaw-mounting of the tissue sections on metabolite stability was also determined. We demonstrate improved stability and biomolecule visualization when funnel-freezing was used to sacrifice the mouse compared with heat-stabilization. Results were further improved when funnel-freezing was combined with fast thaw-mounting of the brain sections.

\section{Keywords:}

Funnel-freezing / Heat-stabilization / Ischemic stroke / MALDI-MSI / Metabolites / Technology

Additional supporting information may be found in the online version of this article at the publisher's web-site
Received: November 3, 2015

Revised: February 17, 2016

Accepted: March 3, 2016
Correspondence: Dr. Liam McDonnell, Center for Proteomics and Metabolomics, Leiden University Medical Center, Einthovenweg 20, 2333 ZC Leiden, The Netherlands

E-mail: I.a.mcdonnell@lumc.nl

\footnotetext{
Abbreviations: ADP, adenosinedifosfaat; AMP, adenosinemonofosfaat; ATP, adenosinetrifosfaat; FF, funnel-freezing; GDP, guanosine diphosphate; GMP, guanosine monophosphate; GTP, guanosine triphosphate; HS, heat-stabilization; MCAo, middle Cerebral Artery occlusion; $\mathbf{M e O H}$, methanol; MSI, mass spectrometry imaging; MSME, multi slice multi echo; NAAG, $\mathrm{N}$-acetylaspartylglutamate; NADH, nicotinamideadeninedinucleotide; T, Tesla; TR, repetition time; TE, echo time
}

\section{Introduction}

MALDI mass spectrometry imaging (MALDI MSI) can simultaneously record the distributions of hundreds of molecules directly from tissue samples [1] and within their histological context [2]. MSI is used to analyse metabolites, drugs, peptides, proteins, lipids and glycans, and is applied to diverse biomedical and biological applications. Tissue preparation is arguably the single most important factor that determines the success of a MALDI MSI experiment, and this is especially true for metabolites on account of their high susceptibility to post-mortem changes [1-3]. For example, for the analysis

\footnotetext{
*I. A. Mulder and C. Esteve contributed equally to this work.
} 


\section{Significance of the study}

Tissue preparation is key to the success of a MALDI MSI experiment as it defines which biomolecular ions may be detected, with which sensitivity, and how they relate to the endogenous levels and distribution. This is especially true of metabolites, which have previously been shown to be prone to rapid post-mortem changes. In this manuscript we demonstrate that funnel-freezing, in combination with rapid tissue mounting, represents a readily implementable, inexpensive manner to acquire metabolite MSI datasets that better retain the original metabolic status than ex-vivo heatbased tissue stabilization. of mouse brain tissue obtained by post-euthanasia freezing, the procedure of decapitation, brain excision and snapfreezing takes one to several minutes, during which time the remaining activity of endogenous enzymes is known to lead to post-mortem degradation [2]. Similar results have also been reported for neuropeptides [4].

Several strategies have been reported for the reduction of post-mortem changes of metabolites in brain tissue:

(i) Heat-stabilization of ex-vivo tissues (HS) - enzymes are inactivated by heating the tissue to $95^{\circ} \mathrm{C}$ using high power heating blocks [2-6]. Blatherwick et al. have demonstrated that ex-vivo heat-stabilization is able to halt the rapid post-mortem degradation of adenine nucleotides that otherwise occurs in ex-vivo snap-frozen tissue [3].

(ii) In-situ freezing (ISF) under anaesthesia is based on freezing the tissue using liquid nitrogen while maintaining blood flow and oxygenation $[2,7,8]$. Hattori et al. have demonstrated that ISF is superior to ex-vivo snapfrozen tissue for maintaining metabolite integrity, including adenine nucleotides that are prone to rapid postmortem change [7].

(iii) In-situ focused microwave irradiation (FMW) - uses focused microwaves to very rapidly, $<2 \mathrm{~s}$, heat the tissue to deactivate enzymes [2]. Sugiura et al. [2] have compared FMW with ISF and ex-vivo snap-freezing and reported that, while ISF and FMW provide similar results for most metabolites, there are several metabolites that are best analysed using FMW on account of their very rapid post-mortem changes [2]. However, the high expense and negative aesthetics of animal sacrifice via focused microwave irradiation has severely limited its use.

ISF and ex-vivo heat-stabilization have been reported to be superior than ex-vivo snap-freezing for preserving metabolic integrity but have not yet been compared. With in-situ funnelfreezing under anaesthesia [9] blood flow is still present until the tissue is frozen. It has previously been demonstrated that warm ischemia times lead to greater post-mortem changes than cold ischemia times [10]. Accordingly, it may be reasoned that in-situ funnel-freezing may better preserve metabolites in their pre-sacrifice state than ex-vivo heat-stabilization, which takes 1-2 $\min$ to excise the brain and another
1-2 min (of warm ischemia) to stabilize the tissue, time which is critical for metabolite stability. A caveat is that with in-situ funnel-freezing enzymes are not inactivated. Accordingly, preparation of the tissue sections for MSI analysis must be very carefully controlled after in-situ funnel-freezing as the enzymes can reactivate, continuing metabolite degradation, as soon as the tissue is thawed [11].

In this paper we have systematically compared in-situ funnel-freezing, ex-vivo heat-stabilization and subsequent thaw-mounting methods for the analysis of metabolites by MALDI-MSI in a mouse model for ischemic stroke. Ischemic stroke is an often disabling event caused by interruption of blood supply to part of the brain [12]. Understanding the biomolecular profiles in the infarct core and penumbra [13] may help explain differential vulnerability and recovery of brain regions to metabolic stress and to search for potential neuroprotective or neurorestorative therapies [14]. In this context, a discriminating factor between core and penumbra is the level of ATP [15]; previous investigations utilizing both bioluminescence and MALDI MSI (using in-situ freezing) have reported a localized increase in ATP. As ATP is very quickly degraded post-mortem, it also represents an excellent model system to assess how well the tissue's metabolic status has been preserved [7].

\section{Materials and methods}

\subsection{Animal protocol}

Male 2- to 4-month-old C57BL/6J mice were used. Experimental stroke was induced using a slightly modified middle cerebral artery occlusion model (MCAo) first described by Longa et al. [16] Mice were anesthetized using isoflurane (3\% induction, $1.5 \%$ maintenance) in 70\% pressurized air and 30\% $\mathrm{O}_{2}$. Carprofen $5 \mathrm{mg} / \mathrm{kg}$, s.c. (Carporal, $50 \mathrm{mg} / \mathrm{mL}$, AST farma B.V., Oudewater, the Netherlands) was given before surgery. During surgery the mouse body temperature was maintained at $37^{\circ} \mathrm{C}$ using a feedback system. Briefly, the surgical procedure; a silicone-coated nylon monofilament (7017PK5Re, Doccol cooperation, Sharon, MA, USA) was introduced into the internal carotid artery, via a small incision in the right common carotid artery, to block the middle cerebral artery at its origin, for $30 \mathrm{~min}$. During the occlusion period, the mouse 
was allowed to wake up in a temperature-controlled incubator (V1200, Peco Services Ltd, Brough, United Kingdom) maintained at $33^{\circ} \mathrm{C}$. After surgery, the animal was placed in the incubator again for $2 \mathrm{~h}$ with easy access to food and water. On a subset of animals, used for the preliminary experiments, SHAM surgery was performed using the same protocol, only without blocking the MCA.

At $24 \mathrm{~h}$ after MCAo mice were scanned in-vivo using a 7-T small animal MRI system (Bruker Pharmascan; Bruker, Ettlingen, Germany), under Paravision 5.1 software (Bruker). A Multi Slice Multi Echo (MSME) sequence protocol was run with TR/TE of $4.000 \mathrm{~ms} / 9 \mathrm{~ms}, 20$ echoes, two averages, matrix $128 \times 128 \mathrm{~mm}$, FOV of $2.50 \mathrm{~cm}$, bandwidth $59523.8 \mathrm{~Hz}$, slice thickness of $0.5 \mathrm{~mm}$ and 16 slices (no gap) and quantitative T2 maps were calculated from the multi-echo trains.

For ex-vivo heat-stabilization the mouse was sacrificed by decapitation and the brain was quickly isolated ( $<1.5 \mathrm{~min}$ ). The brain was immediately stabilized using a tissue heat-stabilizer device (Stabilizor ${ }^{\mathrm{TM}}$, Denator AB, Göteborg, Sweden) at $95^{\circ} \mathrm{C}$ in $60-90 \mathrm{~s}$ depending on brain volume. Thereafter, brains were frozen on dry-ice and stored at $-80^{\circ} \mathrm{C}$.

In-situ funnel-freezing was based on previous reported studies [9, 17-19]. Briefly, the mouse was anesthetized using isoflurane (3\% induction) and 1.5-2\% isoflurane in $30 \% \mathrm{O}_{2}$ and $70 \%$ pressurized air was used to maintain deep anaesthesia. A skin incision was made from the level of the eyes to the occiput exposing the skull. A funnel was placed onto the skull, with the posterior rim of the funnel at the lambdoidal suture. The skin was pulled up around the funnel and secured with four sutures to prevent leaking. Liquid nitrogen was continuously poured into the funnel for $3 \mathrm{~min}$. Thereafter, for easier removal of the brain, the whole animal was frozen in liquid nitrogen. Next the animal was put into dry ice and the brain dissected using a scalpel and a dental drill. The excised and frozen brain was stored at $-80^{\circ} \mathrm{C}$. All animal experiments were approved by the Animal Experiment Ethics Committee of Leiden University Medical Center.

\subsection{MALDI MSI}

Coronal sections $(12 \mu \mathrm{m}$, between -0.10 and +0.40 from Begma) were cut using a cryostat microtome (Leica Microsystems, Wetzlar, Germany) at $-21^{\circ} \mathrm{C}$. The brain sections were thaw-mounted onto ITO glass slides (Delta Technologies, Stillwater, MN, USA) coated with $0.05 \%$ poly-L-lysine (polyL-lysine coating used for greater adherence of tissue sections, protocol used as reported in Aichler et al. [20]). Sections were thaw-mounted onto the slides by localized warming of the reverse side of the MALDI target using a finger for max $3 \mathrm{~s}$ (fast) or for $1 \mathrm{~min}$ (slow) $[1,10]$. The slide-mounted tissues were stored at $-80^{\circ} \mathrm{C}$.

For analysis the slide-mounted tissue sections were first brought to room temperature in a desiccator for $5 \mathrm{~min}$. For matrix application a uniform coating of 9-AA $(2 \mathrm{mg} / \mathrm{mL}$ in $70 \% \mathrm{MeOH}$ ) was added using a SunCollect automated deposition system (SunChrom, Napa, CA, USA). Brain sections from three animals per group were analysed in technical duplicate on a 9.4-Tesla SolariX MALDI-FTICR (Bruker Daltonics, Bremen, Germany), equipped with a SmartBeam II laser system that consists of a frequency tripled Nd:YAG laser operating at $355 \mathrm{~nm}$, at repetition rates up to $1 \mathrm{kHz}$, and using a spatially modulated laser profile. MS data were acquired in negative mode by first accumulating the ions from 500 laser shots in an external hexapole ion trap before transferring them to the ICR cell for detection. Ions were detected in the range $50-1000 \mathrm{~m} / z$ and MSI was performed with a spatial resolution of $125 \mu \mathrm{m}$. Data acquisition, processing, and data visualization were performed using the Flex software suite (FlexControl 3.4, ftmsControl 2.0, FlexImaging 4.1 and DataAnalysis 4.2) from Bruker Daltonics.

After MSI data acquisition the matrix was washed off with $70 \%$ ethanol and the tissue samples stained with cresyl violet (Nissl stain) [21, 22]. High-resolution histological images were obtained with a digital slide scanner (3D Histech MIDI) and were registered to the MSI datasets using FlexImaging. A scheme of the work flow is presented in Fig. 1.

\subsection{Data analysis}

The RMS normalized intensities of six mouse brain sections were measured for each group (two technical duplicates of three biological replicates). MS data were extracted from each MSI dataset for statistical analysis: (1) a non-paired Student's $t$-test (one tailed) was used for comparisons between ex-vivo heat-stabilized and in-situ funnel-freezing brains and (2) a paired Student's $t$-test (one-tailed) was used for comparisons (fast versus slow thaw-mounting) within each mouse brain. Statistical analysis was performed in Microsoft Excel 2010.

Metabolite identities were assigned on the basis of the very high-mass accuracy of the high-field MALDI FTICR mass spectrometer used for the experiments $(<1 \mathrm{ppm})$, in conjunction with the results of previous metabolite MALDI MSI experiments (it is now broadly established that MALDI MSI samples a consistent set of molecules) and the isotope profiles $[2,3,7,8,23,24]$. For selected metabolites, in which the ion intensity was sufficient for MS/MS, the ID's were confirmed by MS/MS.

\section{Results and discussion}

When seeking to investigate the metabolite/peptide content of tissues it is vital that the tissue collection protocol limits the sometimes rapid post-mortem changes that follow animal sacrifice. In a preliminary experiment we compared the metabolite MSI signatures from brain tissue obtained using ex-vivo heat-stabilization and in-situ funnel-freezing with exvivo snap-freezing, to check if the results we obtained were consistent with those previously reported [3,7]; it was indeed found that both methods were more effective at retaining 


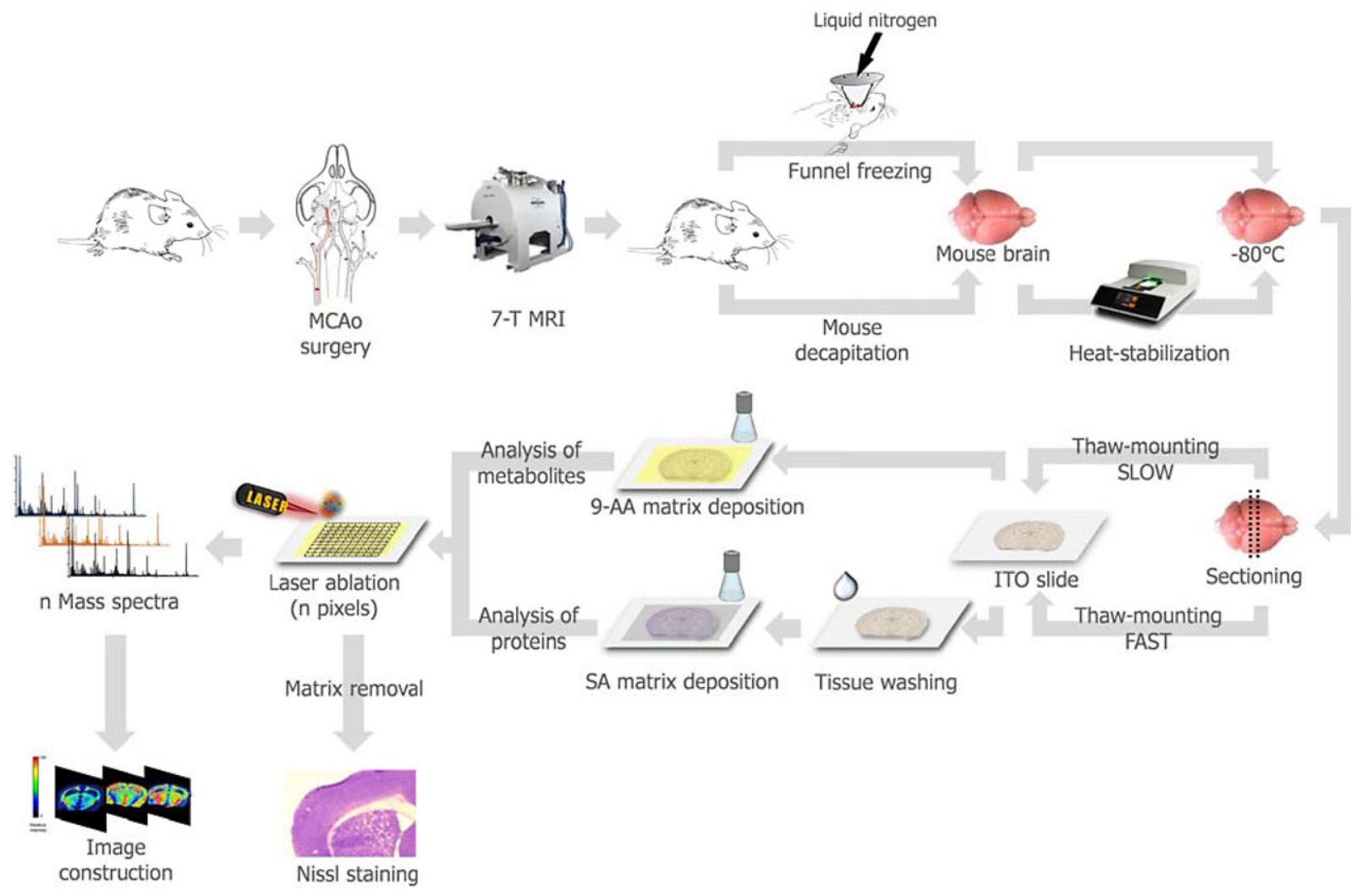

Figure 1. Schematic of the workflow used to analyse the effect of brain tissue sampling protocol. Mice first underwent MCAo surgery, were scanned using a 7.0T MRI at $24 \mathrm{~h}$ and directly thereafter sacrificed by either in-vivo funnel-freezing, or by decapitation followed by ex-vivo heat-stabilization. Coronal tissue sections were then cut and mounted onto poly-lysine coated slides using slow (1 min) or fast ( 3 s) thaw-mounting. Metabolites were analysed by MALDI-FTICR-MSI using 9-AA as the matrix. Each section was stained with Nissl reagent after matrix removal.

labile metabolites but in-situ funnel-freezing appeared to lead to more intense labile metabolite signals (Supporting Information Fig. S1). The results described herein describe an experiment designed to compare ex-vivo heat-stabilization and in-situ funnel-freezing. The comparison included both tissue stabilization methods as well as the time used for thawmounting because in-situ funnel-freezing does not deactivate the tissue enzymes, and thus post-mortem changes may still occur during any subsequent tissue processing step (Fig. 1).

Here we used the MCAo model for ischemic stroke because the localized increase of ATP previously reported by Hattori et al. [7], a molecule highly sensitive to post-mortem degradation [2], provides an excellent in-situ measurement of metabolite stability and the unaffected contralateral hemisphere provides an internal control.

Mouse brain tissue samples were obtained by in-situ funnel-freezing and ex-vivo heat-stabilization, from which $12 \mu \mathrm{m}$ thick coronal tissue sections were placed onto the ITO-coated glass slides using fast $(<3 \mathrm{~s})$ and slow (1 min) thaw-mounting. All experiments were performed in technical duplicate and biological triplicate. Figure 2A shows example MSI images recorded for AMP, ADP, and ATP together with their corresponding T2-weighted MRI and histological im- ages. The localized increase of ATP in the ischemic penumbra was consistently detected in the MSI datasets of the tissues obtained via in-situ funnel-freezing (red circle, Fig. 2A) but not from those obtained via ex-vivo heat-stabilization. Furthermore, it can be seen that when using ex-vivo heat-stabilization the tissue is deformed during the process (Fig. 2A and B, bottom rows) because of the pressure applied to the tissue by the thermal blocks to ensure a high thermal contact. When comparing the MS images with the T2-weighted MR image and the histological section, comparisons are more straightforward using the in-situ funnel-freezing technique.

A significant reduction in AMP, ADP, and ATP signals in the ischemic region was observed for all preparations, in agreement with previous reported results [23]. The ATP signals from the control hemispheres obtained from the exvivo heat-stabilized tissues were lower than those obtained using in-situ funnel-freezing; conversely the AMP signals from the control hemispheres were greater from the exvivo heat-stabilized tissues. Similar results were obtained for the nucleotides GTP, GDP, and GMP as shown in Fig. 2B. However, it is known that MCAo (stroke hemisphere) and heat-stabilization (control hemisphere) can lead to increased salt levels, which can cause a global increase in ionization 

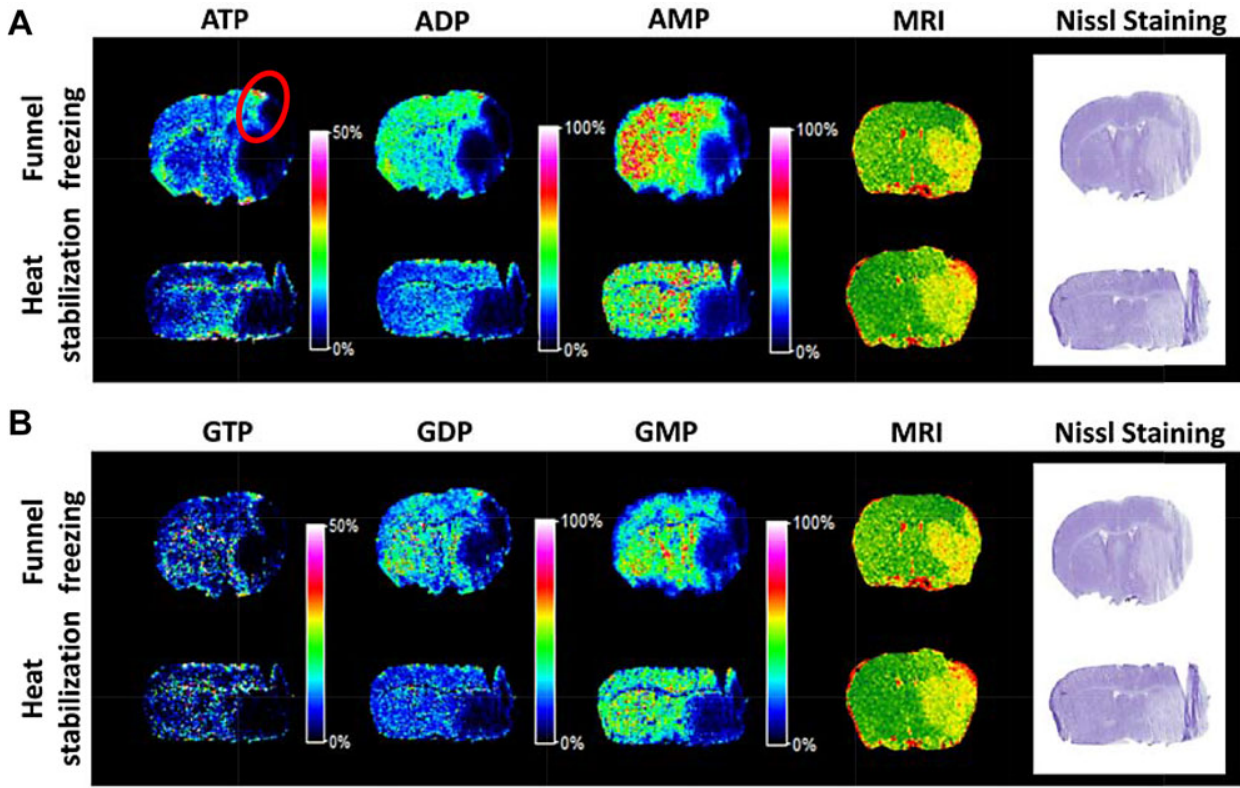

C

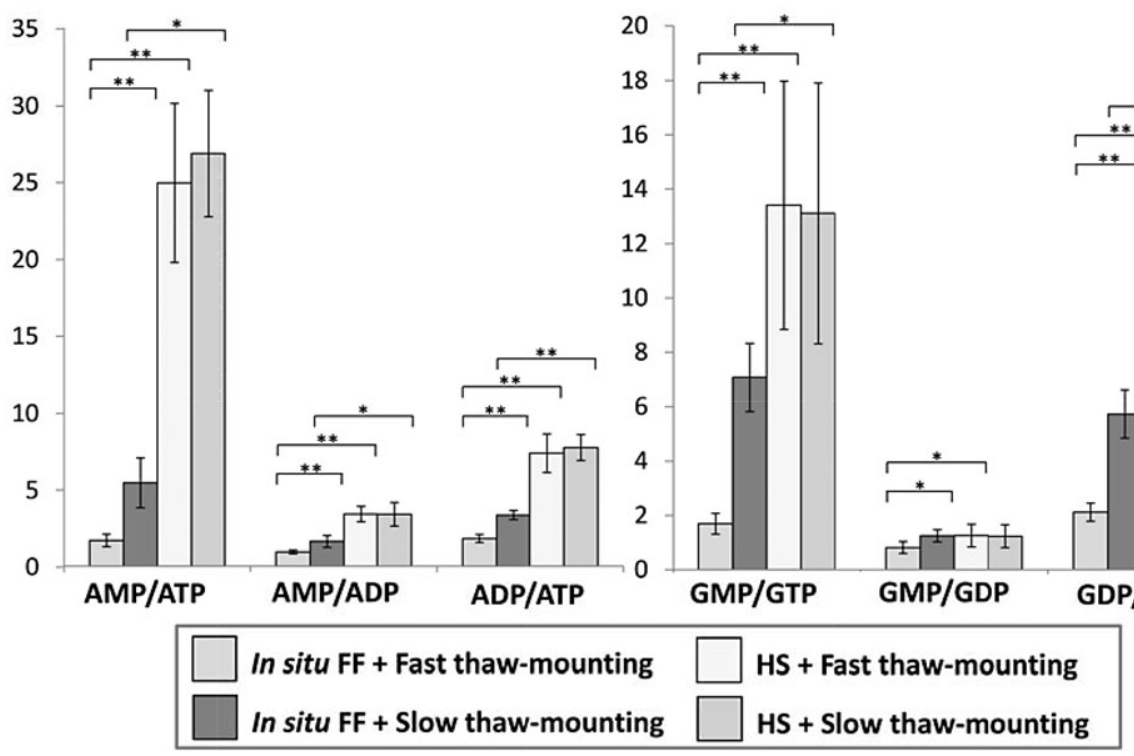

Figure 2. Metabolite MSI dataset showing the effect of the brain tissue sampling protocol on the visualization of adenosine and guanosine metabolites in mouse brain by MALDI-FTICR. (A) Images showing the visualization of the average distribution of AMP $(m / z 346.055)$, ADP $(m / z$ 426.022) and ATP ( $m / z$ 505.988) for mouse brain obtained via ex-vivo heat-stabilization and in-vivo funnel-freezing. (B) Images showing the visualization of the average distribution of GMP $(m / z 362.050)$, GDP $(m / z$ 442.016) and GTP $(m / z 521.982)$ for mouse brain obtained via ex-vivo heat-stabilization and in-vivo funnel-freezing. (C) Average intensity ratios of AMP:ATP, ADP:AMP, ADP:ATP (left) and GMP:GTP, GDP:GMP, GDP:GTP (right) from the control hemisphere, for ex-vivo heat-stabilization (HS) and in-vivo funnel-freezing (FF) with the two thaw-mounting times (slow and fast). Each group consists of three biological replicates each analysed with two technical replicates. Error bars represent standard deviation across technical replicates. ${ }^{* *}=p<0.01$ and $\left.{ }^{*}=p<0.05\right)$ bias and thus lower MSI signal intensities. To circumvent differences in global ionization bias the AMP:ATP, ADP:ATP, and AMP:ADP intensity ratios were calculated (Fig. 2C) from the control hemisphere. The bar chart shows that the ratio of AMP to ATP was 5-fold higher for ex-vivo heatstabilized tissue than for those obtained using in-situ funnelfreezing when slow thaw-mounting was used, and around 15-fold higher when fast thaw-mounting was used. For both thaw-mounting methods (compared with heat-stabilization) the difference in AMP to ATP ratios were statistically significant. Accordingly, the results demonstrate that irrespective of the mounting method used, the in-situ funnel-freezing method leads to less post-mortem changes of the adenine nucleotide metabolites, which is also sup- ported by the observation that the increase in ATP seen in the penumbra was only observed with tissues obtained using in-situ funnel-freezing.

While the in-situ funnel-freezing method was found to lead to reduced post-mortem changes, it is more sensitive to subsequent tissue processing methods. A comparison of the fast and slow thaw-mounting methods on the adenine nucleotide metabolite ratios within the in-situ funnel-freezing brain group revealed a statistically significant increase in metabolite degradation that was not present in the ex-vivo heat-stabilized group, Fig. 2C. This result indicates that for exvivo heat-stabilization all ATP degradation takes place during brain excision and during heat treatment; after heat-induced denaturation the enzymes are no longer active and further 


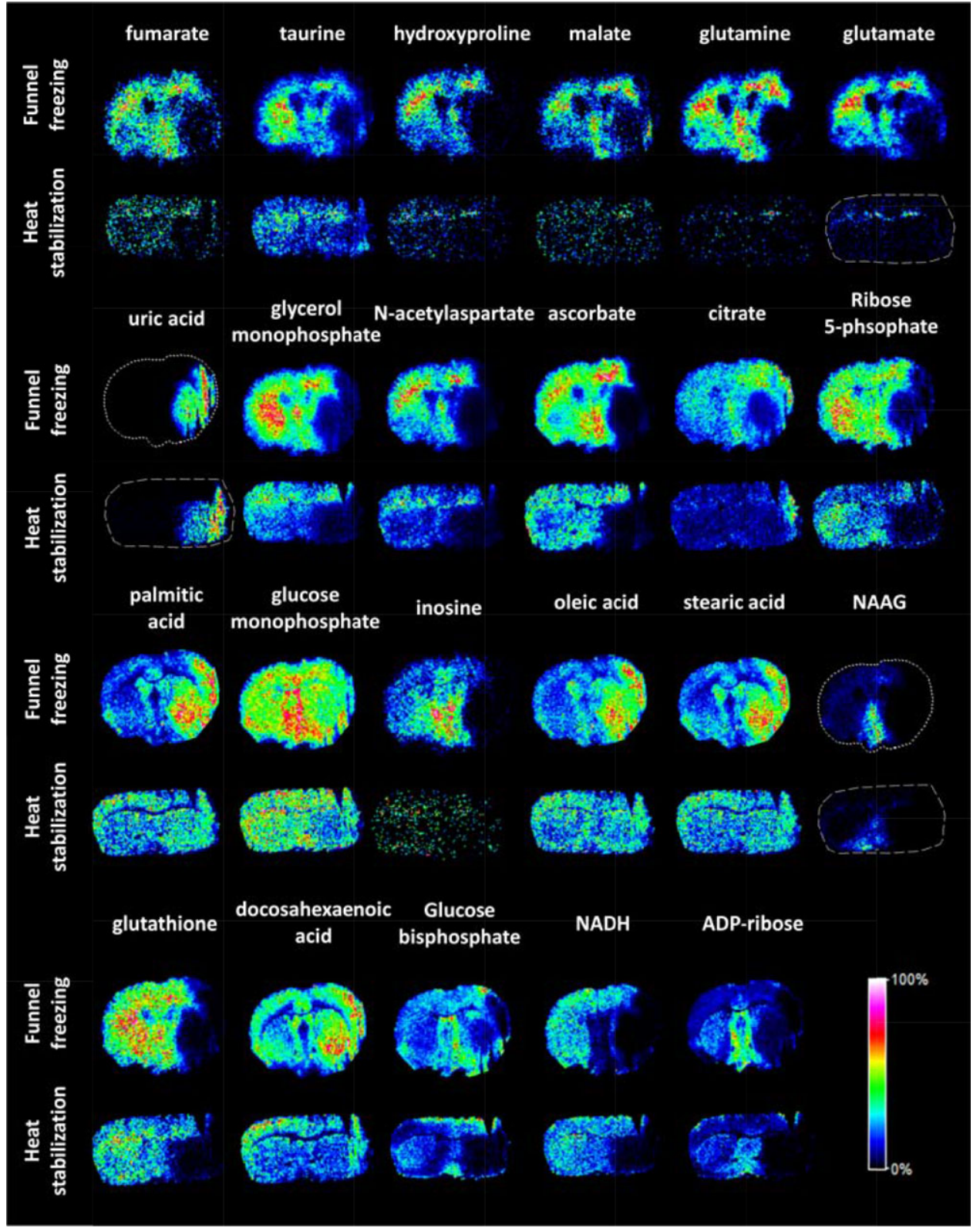

Figure 3. Metabolite MSI images of mouse brainmetabolites found in the range $\mathrm{m} / \mathrm{z}$ 50-1000 and analysed by MALDI-FTICR. metabolite degradation is limited. For in-situ funnel-freezing the enzymes are not deactivated and so once the tissue is thawed post-mortem metabolite degradation may continue. Nevertheless, it should be noted that ATP degradation was always lower when using in-situ funnel-freezing. This is consistent with previous reports that post-mortem changes occur more rapidly when the tissue is still at physiological temperatures $[10,25]$. Similar results were obtained for GTP, GDP and GMP, except for the GMP:GDP ratio (Fig. 2C).

Sample treatment not only influences the stability of adenine nucleotide metabolites. Figure 3 shows the distribution of several metabolites that were detected by MALDI-FTICR-MSI using 9-AA as matrix. In several cases metabolites showed similar distribution for both methods.
However, some of them could only be detected using insitu funnel-freezing or had very weak signal for ex-vivo heat-stabilized brains, including fumarate $(\mathrm{m} / z$ 115.003), hydroxyproline $(m / z 130.050)$, malate $(m / z 133.014)$, glutamine $(m / z 145.061)$, glutamate $(m / z 146.045)$, citrate $(\mathrm{m} / \mathrm{z}$ 191.019), and inosine $(\mathrm{m} / z$ 267.073). Furthermore, the intensity of metabolite peaks were a lot higher when insitu funnel-freezing was used, and also exhibited greater contrast with the stroke region, e.g. taurine $(m / z$ 124.007), glycerol monophosphate $(\mathrm{m} / z$ 171.006), N-acetylaspartate $(m / z 174.040)$, palmitic acid $(m / z 255.232)$, or glutathione $(\mathrm{m} / z$ 306.076).

In their comparison of in-situ freezing, focused microwave irradiation and ex-vivo snap-freezing (no heat-stabilization) 


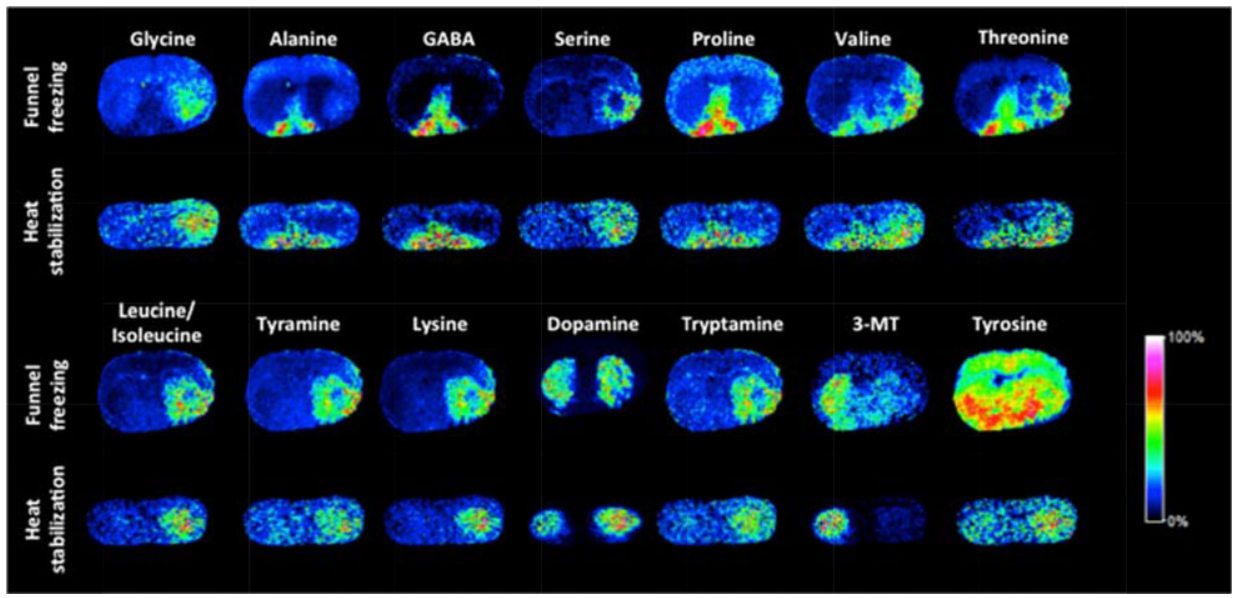

Figure 4. Amino-metabolite MSI images of mouse brain sections analysed using MALDI-FTICR and the on-tissue derivitization agent 2,4-diphenyl-pyranylium tetrafluoro borate [26].
Sugiura et al. [2] used capillary electrophoresis to compare post-mortem metabolite stability. One group, primarily consisting of amino acids, was stable to post-mortem changes. Using the on-tissue derivatization methods recently reported by Shariatgorji et al. [26] we also compared the amino metabolites obtained from tissues processed via in-situ funnelfreezing with those obtained via ex-vivo heat-stabilization, Fig. 4. It can be seen that the distributions are more comparable than those of the less stable metabolites shown in Figs. 2 and 3. Again indicating that the changes are due to post-mortem degradation.

\section{Concluding remarks}

These results again highlight the crucial role of the tissue in MSI experiments, how it was sampled and how it was mounted. Two different tissue sampling strategies, in-situ funnel-freezing and ex-vivo heat-stabilization, as well as fast and slow thaw-mounting, were investigated for their impact on metabolite levels and distributions using MALDI-FTICR-MSI. The results demonstrated that in-situ funnel-freezing has lower degradation compared to heat-stabilization irrespective of the thaw-mounting method, but care should be taken to keep the thaw-mounting short (and thus reproducible) to limit metabolite degradation and variation between experiments. In-situ funnel-freezing has the additional advantage of causing little deformation to the mouse brain, allowing alignment to other imaging datasets and reference tissue atlases.

The authors thank Ludo Broos and Dr. Ricardo Carreira for their assistance with the experimental work; and Dr. Louise van der Weerd for the MRI measurements. This work was supported by the ZonMW Zenith project Imaging Mass SpectrometryBased Molecular Histology: Differentiation and Characterization of Clinically Challenging Soft Tissue Sarcomas (No. 93512002; CE and LMD), Dutch Heart Foundation (2011T055; MJHW), Dutch Brain Foundation (2011(1)-102; MJHW) and ZonMW Veni grant (MJHW), Centre for Medical Systems Bi- ology (CMSB) in the framework of the Netherlands Genomics Initiative (NGI) (AvdM), Marie Curie Career Integration Grant (No. 294233; EAT), FP7 EUROHEADPAIN (No. 602633; AvdM) and Marie Curie IAPP Program BRAINPATH (No. 612360; AvdM, MH \& EAT).

The authors have declared no conflict of interest.

\section{References}

[1] Goodwin, R. J. A., Iverson, S. L., Andren, P. E., The significance of ambient-temperature on pharmaceutical and endogenous compound abundance and distribution in tissues sections when analyzed by matrix-assisted laser desorption/ionization mass spectrometry imaging. Rapid Commun. Mass Spectrom. 2012, 26, 494-498.

[2] Sugiura, Y., Honda, K., Kajimura, M., Suematsu, M., Visualization and quantification of cerebral metabolic fluxes of glucose in awake mice. Proteomics 2014, 14, 829-838.

[3] Blatherwick, E. Q., Svensson, C. I., Frenguelli, B. G., Scrivens, J. H., Localisation of adenine nucleotides in heat-stabilised mouse brains using ion mobility enabled MALDI imaging. Int. J. Mass Spectrom. 2013, 345, 19-27.

[4] Sköld, K., Svensson, M., Norrman, M., Sjögren, B. et al., The significance of biochemical and molecular sample integrity in brain proteomics and peptidomics: stathmin 2-20 and peptides as sample quality indicators. Proteomics 2007, 7, 4445-4456.

[5] Sturm, R. M., Greer, T., Woodards, N., Gemperline, E. et al., Mass spectrometric evaluation of neuropeptidomic profiles upon heat stabilization treatment of neuroendocrine tissues in crustaceans. J. Proteome Res. 2013, 12, 743-752.

[6] Svensson, M., Boren, M., Sköld, K., Fälth, M. et al., Heat stabilization of the tissue proteome: a new technology for improved proteomics. J. Proteome Res. 2009, 8, 974-981.

[7] Hattori, K., Kajimura, M., Hishiki, T., Nakanishi, T. et al., Paradoxical ATP elevation in ischemic penumbra revealed by quantitative imaging mass spectrometry. Antioxid. Redox Signal. 2010, 13, 1157-1167. 
[8] Sugiura, Y., Taguchi, R., Setou, M., Visualization of spatiotemporal energy dynamics of Hippocampal Neurons by mass spectrometry during a kainate-induced seizure. PLoS One 2011, 6, e17952.

[9] Pontén, U., Ratcheson, R. A., Salford, L. G., Siesjö, B. K., Optimal freezing conditions for cerebral metabolites in rats. J. Neurochem. 1973, 21, 1127-1138.

[10] Gündisch, S., Hauck, S., Sarioglu, H., Schott, C. et al., Variability of protein and phosphoprotein levels in clinical tissue specimens during the preanalytical phase. J. Proteome Res. $2012,11,5748-5762$.

[11] Goodwin, R. J., Dungworth, J. C., Cobb, S. R., Pitt, A. R., Time-dependent evolution of tissue markers by MALDI-MS imaging. Proteomics 2008, 8, 3801-3808.

[12] Warlow, C., Sudlow, C., Dennis, M., Wardlaw, J. et al., Stroke. Lancet 2003, 362, 1211-1224.

[13] Hossmann, K. A., Viability thresholds and the penumbra of focal ischemia. Ann. Neurol. 1994, 36, 557-565.

[14] zur Nedden, S., Hawley, S., Pentland, N., Hardie, D. G. et al., Intracellular ATP influences synaptic plasticity in area CA1 of Rat Hippocampus via metabolism to adenosine and activitydependent activation of adenosine A(1) receptors. J. Neurosci. 2011, 31, 6221-6234.

[15] Kohno, K., Hoehn-Berlage, M., Mies, G., Back, T. et al., Relationship between diffusion-weighted MR images, cerebral blood flow, and energy state in experimental brain infarction. Magn. Reson. Imaging 1995, 13, 73-80.

[16] Longa, E. Z., Weinstein, P. R., Carlson, S., Cummins, R., Reversible middle cerebral artery occlusion without craniectomy in rats. Stroke 1989, 20, 84-91.

[17] Fischer, M., Bockhorst, K., Hoehn-Berlage, M., Schmitz, B. et al., Imaging of the apparent diffusion-coefficient for the evaluation of cerebral metabolic recovery after cardiac-arrest. Magn. Reson .Imaging 1995, 13, $781-790$.
[18] Back, T., Hoehn-Berlage, M., Kohno, K., Hossmann, K. A., Diffusion nuclear magnetic resonance imaging in experimental stroke. Correlation with cerebral metabolites. Stroke 1994, 25, 494-500.

[19] Busch, E., Gyngell, M. L., Eis, M., Hoehn-Berlage, M. et al., Diffusion nuclear magnetic resonance imaging in experimental stroke. Correlation with cerebral metabolites. J. Cereb. Blood Flow Metab. 1996, 16, 1090-1099.

[20] Aichler, M., Elsner, M., Ludyga, N., Feuchtinger, A. et al., Clinical response to chemotherapy in oesophageal adenocarcinoma patients is linked to defects in mitochondria. J. Pathol. 2013, 230, 410-419.

[21] Rauser, S., Deininger, S. O., Suckau, D., Hofler, H. et al., Approaching MALDI molecular imaging for clinical proteomic research: current state and fields of application. Expert Rev. Proteomics 2010, 7, 927-941.

[22] Schwamborn, K., Krieg, R. C., Reska, M., Jakse, G. et al., Identifying prostate carcinoma by MALDI-Imaging. Int. J. Mol. Med. 2007, 20, 155-159.

[23] Miura, D., Fujimura, Y., Yamato, M., Hyodo, F. et al., Ultrahighly sensitive in Situ metabolomic imaging for visualizing spatiotemporal metabolic behaviors. Anal. Chem. 2010, 82, 9789-9796.

[24] Benabdellah, F., Touboul, D., Brunelle, A., Laprévote, O., In situ primary metabolites localization on a rat brain section by chemical mass spectrometry imaging. Anal. Chem. 2009, 81, 5557-5560.

[25] Gündisch, S., Annaratone, L., Beese, C., Drecol, E. et al., Critical roles of specimen type and temperature before and during fixation in the detection of phosphoproteins in breast cancer tissues. Lab. Invest. 2015, 95, 561-571.

[26] Shariatgorji, M., Nilsson, A., Goodwin, R. J., Källback, P. et al., Direct targeted quantitative molecular imaging of neurotransmitters in brain tissue sections. Neuron 2014, 84, 697-707. 